\title{
Down-Regulation of Claudin-1 Expression in Gastric Cancer Mucosa Is Correlated with Poor Prognosis
}

\author{
Eiichiro Hirakawa1 ${ }^{*}$, Yasunori Tokuhara1 ${ }^{1}$, Tatsuya Morinishi ${ }^{1}$, Hiroyuki Ohsaki ${ }^{2}$, \\ Erika Uemura ${ }^{3}$, Yukari Miki", Toru Matsunaga ${ }^{3}$, Yoshio Kushida ${ }^{3}$, Reiji Haba ${ }^{3}$ \\ ${ }^{1}$ Laboratory of Pathology, Department of Medical Technology, Kagawa Prefectural University of Health \\ Sciences, Kagawa, Japan \\ ${ }^{2}$ Department of Medical Technology, Ehime Prefectural University of Health Sciences, Ehime, Japan \\ ${ }^{3}$ Department of Diagnostic Pathology, Kagawa University Hospital, Kagawa, Japan \\ ${ }^{4}$ Department of Medical Hygiene Technology Course, Kochi Gakuen College, Kochi, Japan \\ Email: ${ }^{*}$ hirakawa@chs.pref.kagawa.jp
}

Received 27 September 2014; revised 10 October 2014; accepted 20 October 2014

Copyright (C) 2014 by authors and Scientific Research Publishing Inc.

This work is licensed under the Creative Commons Attribution International License (CC BY).

http://creativecommons.org/licenses/by/4.0/

(c) (i) Open Access

\section{Abstract}

Background: Cell adhesion molecule abnormalities are given as one reason for the occurrence of invasion and metastasis in various cancers. In this study, we conducted an immunohistochemical examination of cell adhesion molecule claudin-1 in mucosa and invasive front of gastric cancer, and investigated the correlation of claudin-1 expression and clinicopathological factors. Methods: Immunohistochemical examination was performed for 35 patients who underwent surgery between January 2010 and October 2011, to assess the correlation of claudin-1 with primary gastric cancer. Results: The expression rate of claudin-1 was $48.6 \%(17 / 35)$ in 35 gastric carcinoma patients. The positive rates of claudin-1 were $42.9 \%(15 / 35)$ in mucosa and $28.6 \%(10 / 35)$ in invasive front of gastric cancer. The expression rate of claudin-1 in invasive front was lower than in mucosa. From comparing claudin-1 expression in mucosa, it was found that well-to moderately-differentiated adenocarcinoma had significantly more claudin-1 expression than poorly-differrentiated adenocarcinoma. Claudin-1 expression of well-to moderately-differentiated adenocarcinoma decreased in the invasive front of gastric cancer. Expression of claudin-1 in mucosa was negative in many cases with advanced tumor invasion (T2, T3, T4) and positive in many cases with early tumor invasion (T1), with a significant difference between the two $(p<0.05)$. In mucosa, many cases at an advanced stage (Stage II, III, IV) were negative for claudin-1 expression and many cases at an early stage (Stage I) were positive for claudin-1 expression. A significant difference was seen between the two $(p<0.05)$. However, claudin-1 expression in the invasive front was not cor-

\footnotetext{
"Corresponding author.
} 
related with histological type, depth of tumor invasion and stage. Conclusion: From the above results, it is considered that decreased claudin-1expression in mucosa is related to histological type, gastric cancer invasion and stage, and this information may be useful when making a pathological diagnosis of advanced gastric cancer and estimating outcomes.

\section{Keywords}

Claudin-1, Immunohistochemistry, Mucosa, Invasive Front, Gastric Cancer

\section{Introduction}

Gastric carcinoma is one of the most frequent malignant tumors and the second most common cause of death from carcinoma in the world, in spite of improvements and development in endoscopy and surgical technology [1]-[3]. The extent of cancer invasion and metastasis are critical events for deciding on a cancer treatment, and for predicting a prognosis.

Tight junctions are the most apical of cellular junctions, providing a selective barrier and maintaining cellular polarity in epithelial cells [4]-[6]. Abnormality of tight junction proteins or adherence junction proteins, such as claudins, $\beta$-catenin and E-cadherin, is considered to be an important pathway and trigger for tumor progression and metastasis [7]-[10]. Among the many tight junction proteins, the four-time transmembrane protein family, claudins, are studied in several malignancies, such as those of the breast, ovary, uterus, urinary bladder, thyroid, pancreas, colon and stomach [11] [12]. The expression of different claudins has been shown in several tumors including gastric cancer.

The expression of claudins- 1 is reportedly lower in cases of diffuse typegastric carcinoma than in intestinal type gastric carcinoma [11]. On the other hand, the expression of claudin-1 is significantly lower in cases of intestinal type rather than diffuse type gastric carcinoma [13]. Previous studies have demonstrated the heterogeneities of claudin immunoreactivities in gastric carcinoma. In addition, there have been a few reports regarding claudin-1 expression in mucosa and the invasive front, comparing the clinicopathological factors in the early and advanced stage of gastric cancer [14]. In the study, the claudin-1 over-expression rate in the invasive front was positively related with the differentiation, invasiveness and metastasis of gastric carcinoma. However, the exact role of claudin-1 protein in gastric cancer remains controversial and unclear. In particular, the biological function of claudins in mucosa and the invasive front has not been clarified. In this study, we aimed to evaluate the expression of claudin-1 in mucosa and invasive front of gastric cancer, and investigate the correlation of claudin-1expression and clinicopathological factors. We found that the expression of claudin- 1 in mucosa showed a significant correlation with the histological type. The expression of claudin-1 in mucosa was also significantly correlated with advanced T-stage and advanced staging, as opposed to early T-stage and early stage.

\section{Materials and Methods}

\subsection{Patients and Material}

Tissues were obtained from surgically resected specimens of 35 patients, diagnosed as gastric carcinoma between January 2010 and October 2011 in Kagawa University Hospital, for claudin-1 immunohistochemistry. The histological findings, lymph node metastases, venous invasion and TNM stage were evaluated based on the Fourteenth Japanese Classification of Gastric Adenocarcinoma and the Seventh Union for International Cancer Control (UICC) TNM Staging System [15]-[17]. As for the histological type of adenocarcinoma, the major type that occupied the most extensive area was defined as the histological type for a case. In this study, the term "invasive front" was defined as the transitional area between the primary cancer and normal interstitium. All subjects signed a written informed consent form approved by the hospital. The study was conducted with the approval of the Institute Research Ethics Committee of Kagawa Prefectural University of Health Sciences.

\subsection{Immunohistochemistry}

Specimens were fixed in formalin, embedded in paraffin wax, cut into $4 \mu \mathrm{m}$ thick sections and stained with he- 
matoxylin and eosin. The sections were immunostained with antibodies for monoclonal mouse anti-claudin 1 (1:200, Abcam, Cambridge, UK). The sections were deparaffinized and heated in an autoclave for $15 \mathrm{~min}$ in $0.01 \mathrm{M}$ citrate buffer ( $\mathrm{pH}$ 6.0) to retrieve the antigens. Endogenous peroxidase activity was blocked with the use of 3\% hydrogen peroxide for $10 \mathrm{~min}$. The sections were incubated with primary antibodies for 2 hours at room temperature with secondary antibodies of Histofine Simple Stain MAX PO (MULTI) (universal immuno-peroxidase polymer, anti-mouse and anti-rabbit) according to the manufacturer's instructions (Nichirei Biosciences, Tokyo, Japan), and then immersed into diaminobenzidine tetrahydrochloride (DAB) solution (Nichirei Biosciences, Tokyo, Japan). The sections were counterstained with hematoxylin, dehydrated and mounted. As positive controls, normal gastric mucosa samples were used.

The classification category of claudin expression was based on the criteria of Jung et al. [13]. Briefly, the immunostaining for claudin- 1 was assessed as follows at the mucosa and invasive front, respectively. The scores were 0 , no staining; $1+,<25 \%$ cells positive and incomplete membranous staining; $2+, 25 \%-50 \%$ cells positive and incomplete membranous staining; $3+, 50 \%-75 \%$ cells positive and complete or incomplete membranous staining; $4+,>75 \%$ cells positive and complete membranous staining. In the evaluation, the expression of claudin- 1 was grouped into negative $\left(0,1^{+}\right)$and positive $\left(2+, 3^{+}, 4^{+}\right)$levels.

\subsection{Statistics}

Univariate analysis was performed using the chi-square test or Fisher's exact test for categorical data. All statistical analyses were performed with SPSS Ver. 22.0. The statistical comparative analysis was performed by chi-square test, and $\mathrm{P}$ values were considered significant if $<0.05$.

\section{Results}

\subsection{Clinicopathological Parameters}

Table 1 summarizes the clinicopathological parameters of 35 patients with gastric adenocarcinoma having undergone immunohistochemistry for claudin-1. Twenty-four patients (68.6\%) were men and $11(31.4 \%)$ patients were women, with a median age of 71.7 years old (age range, 59 - 91 y). Fifteen (42.9\%) patients had stage I, 6 (17.1\%) patients had stage II, 7 (20.0\%) patients had stage III, and 7 (20.0\%) patients had stage IV adenocarcinoma.

\subsection{Expression of Claudin-1 in Mucosa and Invasive Front}

Claudin-1 was mainly expressed in the cell membrane of gastric adenocarcinoma cells and some samples displayed a low level of cytoplasmic staining. The expression rate of claudin-1 was $48.6 \%$ (17/35) in 35 gastric carcinoma patients. Claudin-1 expression in mucosa and invasive front was compared in 35 gastric adenocarcinoma patients. The expression of claudin- 1 was $42.9 \%$ (15/35) in mucosa and $28.6 \%(10 / 35)$ in invasive front (Figure 1). The expression of claudin-1 in mucosa was significantly higher in well- to moderately-differentiated adenocarcinomas than in poorly-differentiated adenocarcinomas $(p<0.05)$. The expression of claudin-1 in mucosa was significantly lower in cases of advanced depth tumor invasion (T2, T3, T4) and advanced stage (Stage II, III, IV), than in cases of mucosal invasion (T1) and early stage (Stage I). The expression of claudin-1 in mucosa was significantly related with the extent of tumor invasion depth and TNM staging. The expression of claudin-1 in mucosa was not related with lymphatic invasion, venous invasion and lymph node metastasis. The expression of claudin-1 in invasive front was not related with histological differentiation, lymphatic invasion, venous invasion, lymph node metastasis, depth of tumor invasion and staging (Table 2).

\section{Discussion}

In this study, we conducted immunohistochemical examinations of cell adhesion protein molecules, expression of claudin-1 in mucosa and invasive front of gastric carcinoma patients, although the sample size was small compared with other claudin studies. Our results revealed that the expression of claudin- 1 in mucosa was significantly higher in well- to moderately-differentiated adenocarcinoma than in poorly-differentiated adenocarcinoma. The expression of claudin-1 in well- to moderately-differentiated adenocarcinoma and poorly-differentiated adenocarcinoma was decreased in the invasive frontrather than in mucosa. The expression of claudin-1 in inva- 
Table 1. Clinicopathological characteristics for Claudin-1study $(\mathrm{n}=35)$.

\begin{tabular}{|c|c|}
\hline Basic characteristics & Number of patients (\%) \\
\hline Age (mean \pm SD) (yr) & $71.7 \pm 8.2$ \\
\hline \multicolumn{2}{|l|}{ Gender } \\
\hline Male & $24(68.6)$ \\
\hline Female & $11(31.4)$ \\
\hline \multicolumn{2}{|l|}{ Histological type } \\
\hline Well/moderately differentiated & 15 (42.9) \\
\hline Poorly-differentiated & $20(57.1)$ \\
\hline \multicolumn{2}{|l|}{ Lymphatic invasion } \\
\hline Negative & $16(45.7)$ \\
\hline Positive & $19(54.3)$ \\
\hline \multicolumn{2}{|l|}{ Venous invasion } \\
\hline Negative & $9(25.7)$ \\
\hline Positive & $26(74.3)$ \\
\hline \multicolumn{2}{|l|}{ Lymph node metastasis } \\
\hline No & $16(45.7)$ \\
\hline N1 & $8(22.9)$ \\
\hline N2 & $1(2.9)$ \\
\hline N3 & $10(28.6)$ \\
\hline \multicolumn{2}{|l|}{ Depth of tumor invasion } \\
\hline $\mathrm{T} 1$ & $11(31.4)$ \\
\hline $\mathrm{T} 2$ & $4(11.4)$ \\
\hline T3 & $8(22.9)$ \\
\hline $\mathrm{T} 4$ & $12(34.3)$ \\
\hline \multicolumn{2}{|l|}{ Stage } \\
\hline I & 15 (42.9) \\
\hline II & $6(17.1)$ \\
\hline III & $7(20.0)$ \\
\hline IV & $7(20.0)$ \\
\hline
\end{tabular}

sive front was also unrelated with histological differentiation, lymphatic invasion, venous invasion, lymph node metastasis, depth of tumor invasion and staging. However, the expression of claudin-1 in mucosa was significantly lower for an advanced T-stage (T2, T3, T4) and advanced staging (Stage II, III, IV) compared with an early T-stage (T1) and early stage (Stage I) (Table 2). These results showed that the expression of claudin-1 was different in each area of the mucosa and invasive front in gastric carcinoma. Our results showed that down-regulation of claudin-1 expression had an important role in gastric cancer invasion.

Claudins are important proteins in cellular tight junctions that maintained barrier formation, cell polarity, signal transduction and cell proliferation. Dysregulation of claudins has been reported in many different cancers, such as those originating from the breast, liver, esophagus, pancreas, colon, and stomach [11] [12]. Previous studies have reported about the loss or down-regulation of tight junction proteins in cancer cells [18] [19]. 
Table 2. Correlation between Claudin-1 expression and clinicopathologic characteristics $(\mathrm{n}=35)$.

\begin{tabular}{|c|c|c|c|c|c|c|}
\hline & \multicolumn{3}{|c|}{ Mucosa } & \multicolumn{3}{|c|}{ Invasive front } \\
\hline & $(-)$ & $(+)$ & $p$-value & $(-)$ & $(+)$ & $p$-value \\
\hline \multicolumn{7}{|l|}{ Histological type } \\
\hline Well/moderately differentiated & 5 & 10 & 0.034 & 8 & 7 & 0.062 \\
\hline Poorly-differentiated & 15 & 5 & & 17 & 3 & \\
\hline \multicolumn{7}{|l|}{ Lymphatic invasion } \\
\hline Negative & 9 & 7 & 0.807 & 9 & 7 & 0.132 \\
\hline Positive & 11 & 8 & & 16 & 3 & \\
\hline \multicolumn{7}{|l|}{ Venous invasion } \\
\hline Negative & 3 & 6 & 0.129 & 4 & 5 & 0.099 \\
\hline Positive & 17 & 9 & & 21 & 5 & \\
\hline \multicolumn{7}{|l|}{ Lymph node metastasis } \\
\hline No & 9 & 7 & 0.807 & 9 & 7 & 0.132 \\
\hline N1, N2, N3 & 11 & 8 & & 16 & 3 & \\
\hline \multicolumn{7}{|l|}{ Depth of tumor invasion } \\
\hline $\mathrm{T} 1$ & 3 & 8 & 0.027 & 5 & 6 & 0.057 \\
\hline $\mathrm{T} 2, \mathrm{~T} 3, \mathrm{~T} 4$ & 17 & 7 & & 20 & 4 & \\
\hline \multicolumn{7}{|l|}{ Stage } \\
\hline I & 5 & 10 & 0.034 & 8 & 7 & 0.062 \\
\hline II, III, IV & 15 & 5 & & 17 & 3 & \\
\hline
\end{tabular}
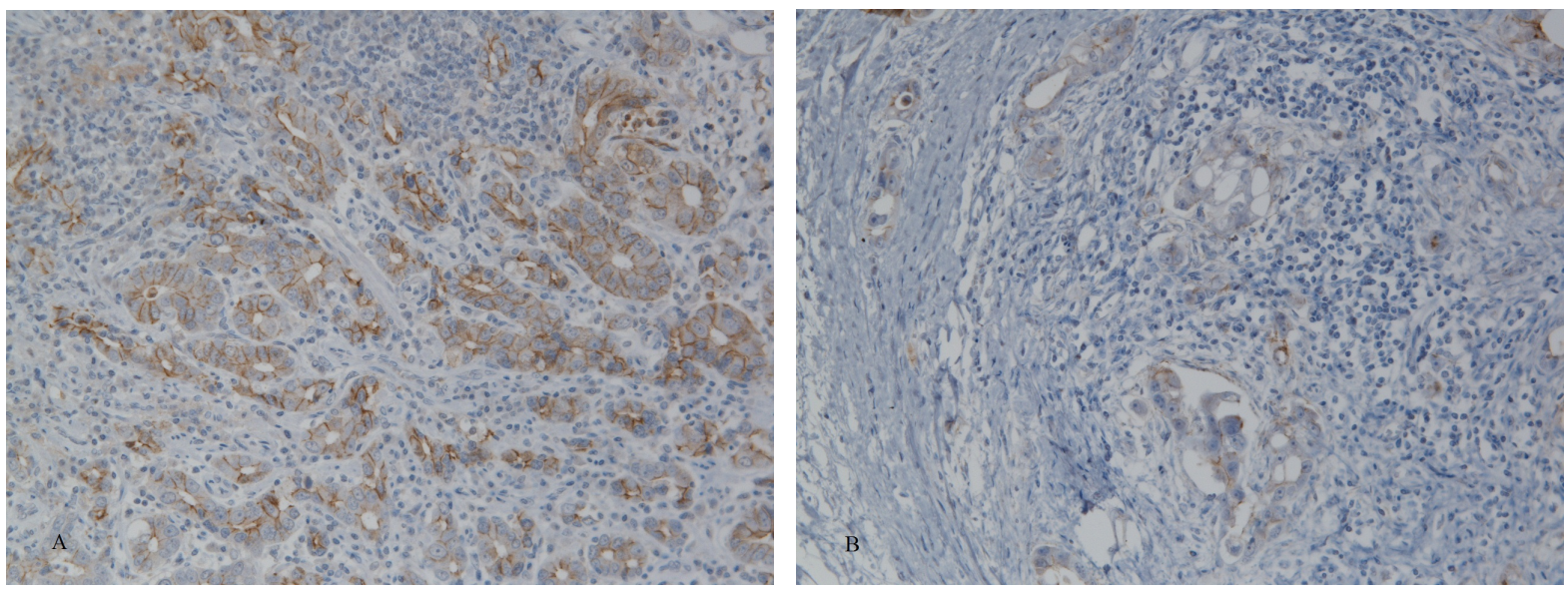

Figure 1. Immunohistochemical staining showing positive level (4+) of claudin-1 in mucosa (A) and negative level (1+) in invasive front (B) of gastric cancer $(\times 200)$.

Claudin-1 has been found to be reduced in breast cancer as well as colon cancer [20]-[22]. The expression of claudin-1 and its clinicopathological correlation has been frequently reported for gastric cancer. Soini et al. [11] have reported that claudin- 1 expression is $53.6 \%$, that the expression of claudin- 1 is lower in diffuse type than intestinal type, and that the expression of claudin-1, 3, 4 and 5 had an important role in determining the phenotype and loose cohesion of cells in the diffuse type of gastric cancer. Resnick et al. [23] demonstrated decreased claudin-1 expression in the diffuse type compared to the intestinal type of gastric cancer. Histologically, gastric adenocarcinomas can be divided into differentiated and undifferentiated adenocarcinomas, including poorly-differentiated adenocarcinoma. They were also classified as intestinal type and diffuse type, or expanding and infiltrative type [24] [25]. Generally, intestinal type is better differentiated with cohesive, glandular-like tumor cells, whereas diffuse type is poorly differentiated with infiltration and non-cohesive tumor cells. Thus, our re- 
sults were consistent with theirs. Jung et al. [13] reported that the expression of claudin-3 was significantly lower in cases with an advanced T-stage (T3 and T4) of gastric adenocarcinoma. In that study, the expression rate of claudin-1 was $56.9 \%$ in gastric adenocarcinoma, and the claudin-1 expression was significantly lower in the M1 stage (distant metastasis) compared with the M0 stage (no distant metastasis) (27.3\% vs. $62.3 \%$ ). The reduction of claudins in cancer is consistent with the concept of tumorigenesis, accompanied by a disruption of tight junctions. The loss or low expression of claudins in cancer cells leads to loss of cell adhesion, histological differentiation, and the progression of cancer to metastasis. Our results showed that the expression of claudin- 1 in mucosa was significantly lower for advanced T-stage and advanced staging. This may show that disruption of claudin-1 is associated with carcinogenesis and progression of gastric cancer. These results indicate that claudin-1 may play a role in determining the diffuse phenotype and loose cohesion of cells in diffuse-type gastric carcinoma in a manner similar to that of E-cadherin [11].

Our results showed the expression of claudin-1 in mucosa was significantly higher in well- to moderatelydifferentiated adenocarcinoma than in poorly-differentiated adenocarcinoma. In contrast, Wu et al. [14] reported that claudin-1 expression in invasive front was different from that in the mucosa of gastric cancer, and that the claudin-1 up-regulation in invasive front was positively related with the differentiated degree, and with the invasiveness and metastasis of gastric adenocarcinoma. Huang et al. [26] showed that claudin-1 expression was increased in tumor tissues that were of intestinal type, differentiated type, at an advanced TNM stage and with lymph node metastasis. Their results are consistent with our findings which showed loss of claudin-1 in diffuse type gastric cancer. However, the prognostic significance of claudin-1 in their study was different from that of our study and previous reports [11] [23]. They suggested that the difference in prognostic significance from the previous reports may have been caused by the difference in patient nationality. In addition, Eftung et al. [27] demonstrated that the claudin-1 gene was significantly up-regulated in gastric cancer compared to matched normal mucosa, and that claudin-1 expression was correlated with reduced survival. These results were different from those of our study, however, based on biology, the down-regulation of claudin-1 would result in destruction of tight junctions and loss of cell-to-cell adhesion causing tumor progression [28] [29]. Thus, the expression pattern of claudin- 1 in gastric cancer was diverse and controversial. This may have been caused by the different immunohistochemistry scoring systems of the reported studies. But the mechanisms for the up-regulation or down-regulation of claudin-1 remains incompletely understood, and the role of claudin-1 in cancer progression and progress is far from clear. These paradoxical issues require further investigation.

In conclusion, there have been few reports comparing expression of claudin-1 in mucosa and the invasive front of gastric cancer. Down-regulation of claudin-1 expression in mucosa correlates with poor prognostic factors, according to pathological findings. These findings indicate that the perturbation of claudin- 1 has an important role in the progression of gastric cancer. However, the subjects are few in this study, and further investigations with a larger sample are required to better identify the claudin-1 action mechanism in gastric cancer.

\section{References}

[1] Kang, C., Song, J., Lee, K. and Kim, M.Y. (2014) Epigenetics: An Emerging Player in Gastric Cancer. World Journal of Gastroenterology, 20, 6433-6447. http://dx.doi.org/10.3748/wjg.v20.i21.6433

[2] Yamamoto, S. (2001) Stomach Cancer Incidence in the World. Japanese Journal of Clinical Oncology, 31, 471.

[3] Ferlay, J., Shin, H.R., Bray, F., et al. (2010) Estimates of Worldwide Burden of Cancer in 2008: GLOBOCAN 2008. International Journal of Cancer, 127, 2893-2917. http://dx.doi.org/10.1002/ijc.25516

[4] Matter, K. and Balda, M.S. (2003) Signalling to and from Tight Junctions. Nature Reviews Molecular Cell Biology, 4, 225-236. http://dx.doi.org/10.1038/nrm1055

[5] Mitic, L.L., Van Itallie, C.M. and Anderson, J.M. (2000) Molecular Physiology and Pathophysiology of Tight junctions I. Tight Junction Structure and Function: Lessons from Mutant Animals and Proteins. American Journal of Physiology-Gastrointestinal and Liver Physiology, 279, 250-254.

[6] Tsukita, S., Furuse, M. and Itoh, M. (2001) Multifunctional Strands in Tight Junctions. Nature Reviews Molecular Cell Biology, 2, 285-293. http://dx.doi.org/10.1038/35067088

[7] Martin, T.A. and Jiang, W.G. (2001) Tight Junctions and Their Role in Cancer Metastasis. Histology and Histopathology, 16, 1183-1195.

[8] Langbein, L., Pape, U.F., Grund, C., et al. (2003) Tight Junction-Related Structures in the Absence of a Lumen: Occludin, Claudins and Tight Junction Plaque Proteins in Densely Packed Cell Formations of Stratified Epithelia and Squamous Cell Carcinomas. European Journal of Cell Biology, 82, 385-400. http://dx.doi.org/10.1078/0171-9335-00330 
[9] Jawhari, A., Jordan, S., Poole, S., Browne, P., Pignatelli, M. and Farthing, M.J. (1997) Abnormal Immunoreactivity of the E-Cadherin-Catenin Complex in Gastric Carcinoma: Relationship with Patient Survival. Gastroenterology, 112, 46-54. http://dx.doi.org/10.1016/S0016-5085(97)70218-X

[10] Mimata, A., Fukamachi, H., Eishi, Y. and Yuasa Y. (2011) Loss of E-Cadherin in Mouse Gastric Epithelial Cells Induces Signet Ring-Like Cells, a Possible Precursor Lesion of Diffuse Gastric Cancer. Cancer Science, 102, 942-950. http://dx.doi.org/10.1111/j.1349-7006.2011.01890.x

[11] Soini, Y., Tommola, S., Helin, H. and Martikainen, P. (2006) Claudins 1,3,4 and 5 in Gastric Carcinoma, Loss of Claudin Expression Associates with the Diffuse Subtype. Virchows Archiv, 448, 52-58. http://dx.doi.org/10.1007/s00428-005-0011-6

[12] Hewitt, K.J., Agareal, R. and Morin, P.J. (2006) The Claudin Gene Family: Expression in Normal and Neoplastic Tissues. BMC Cancer, 6, 186.

[13] Jung, H., Jun, K.H., Jung, J.H., Chin, H.M. and Park, W.B. (2011) The Expression of Claudin-1, Claudin-2, Claudin-3, and Claudin-4 in Gastric Cancer Tissue. Journal of Surgical Research, 167, 185-191. http://dx.doi.org/10.1016/j.jss.2010.02.010

[14] Wu, Y.L., Zhang, S., Wang, G.R. and Chen, Y.P. (2008) Expression Tranformation of Claudin-1 in the Process of Gastric Adenocarcinoma Invasion. World Journal of Gastroenterology, 14, 4943-4948. http://dx.doi.org/10.3748/wjg.14.4943

[15] Japanese Gastric Cancer Association (2011) Japanese Classification of Gastric Gastric Carcinoma: 3rd English Edition. Gastric Cancer, 14, 101-112. http://dx.doi.org/10.1007/s10120-011-0041-5

[16] Sano, T. and Aiko, T. (2011) New Japanese Classifications and Treatment Guidelines for Gastric Cancer: Revision Concepts and Major Revised Points. Gastric Cancer, 14, 97-100. http://dx.doi.org/10.1007/s10120-011-0040-6

[17] Sobin, L.H., Gospodarowicz, M.K. and Wittekind, C., Eds. (2007) TNM Classification of Malignant Tumours. 7th Edition, John Wiley \& Sons, New York.

[18] Jechlinger, M., Grunert, S. Tamir, I.H., Janda, E., Lüdemann, S., Waerner, T., et al. (2003) Expression Profiling of Epithelial Plasticity in Tumor Progression. Oncogene, 22, 7155-7169. http://dx.doi.org/10.1038/sj.onc.1206887

[19] Tsukita, S. and Furuse, M. (2000) Pores in the Wall: Claudins Constitute Tight Junction Strands Containing Aqueous Pores. The Journal of Cell Biology, 149, 13-16. http://dx.doi.org/10.1083/jcb.149.1.13

[20] Resnick, M.B., Konkin, T., Routhier, J., Sabo, E. and Pricolo, V.E. (2005) Claudin-1 is a Strong Prognostic Indicator in Stage II Colonic Cancer: A Tissue Microarray Study. Modern Pathology, 18, 511-518. http://dx.doi.org/10.1038/modpathol.3800301

[21] Krämer, F., White, K., Kubbies, M., Swisshelm, K. and Weber, B.H.F. (2000) Genomic Organization of Claudin-1 and Its Assessment in Hereditary and Sporadic Breast Cancer. Human Genetics, 107, 249-256. http://dx.doi.org/10.1007/s004390000375

[22] Tökés, A.M., Kulka, J., Paku, S., Szik, Á., Páska, C., Novák, P.K., et al. (2005) Claudin-1, -3 and -4 Proteins and mRNA Expression in Benign and Malignant Breast Lesions: A Research Study. Breast Cancer Research, 7, R296R305. http://dx.doi.org/10.1186/bcr1265 http://dx.doi.org/10.1186/bcr983

[23] Resnick, M.B., Gavilannez, M., Newton, E., Konkin, T., Bhattachrya, B., Britt, D., et al. (2005) Claudin Expression in Gastric Adenocarcinomas: A Tissue Microarray Study with Prognostic Correlation. Human Pathology, 36, 886-892. http://dx.doi.org/10.1016/j.humpath.2005.05.019

[24] Lauren, P. (1965) The Two Histological Main Types of Gastric Carcinoma: Diffuse and So-Called Intestinal-Type Carcinoma. An Attempt at a Histo-Clinical Classification. Acta Pathologica et Microbiologica Scandinavica, 64, 31-49.

[25] Ming, S.C. (1997) Gastric Carcinoma. A Pathobiological Classification. Cancer, 39, 2475-2485.

[26] Huang, J., Li, J., Qu, Y., Zhang, J., Zhang, L., Chen, X., et al. (2014) The Expression of Claudin 1 Correlates with $\beta$ Catenin and Is a Prognostic Factor of Poor Outcome in Gastric Cancer. International Journal of Oncology, 44, 12931301.

[27] Eftang, L.L., Esbensen, Y., Tannes, T.M., Blom, G.P., Bunkholm, I.R. and Bunkholm, G. (2013) Up-Regulation of CLDN1 in Gastric Cancer is Correlated with Reduced Survival. BMC Cancer, 13, 586.

[28] Ohtani, S., Terajiima, M., Satoh, J., Soeta, N., Saze, Z., Kashimura, S., et al. (2009) Expression of Tight-Junction-Associated Proteins in Human Gastric Cancer: Downregulation of Claudin-4 Correlates with Tumor Aggressiveness and Survival. Gastric Cancer, 12, 43-51. http://dx.doi.org/10.1007/s10120-008-0497-0

[29] Gao, M., Li, W., Wang, H. and Wang, G. (2013) The Distinct Expression Patterns of Claudin-10, -14, -17 and E-Cadherin between Adjacent Non-Neoplastic Tissues and Gastric Cancer Tissues. Diagnostic Pathology, 8, 205. http://dx.doi.org/10.1186/1746-1596-8-205 
Scientific Research Publishing (SCIRP) is one of the largest Open Access journal publishers. It is currently publishing more than 200 open access, online, peer-reviewed journals covering a wide range of academic disciplines. SCIRP serves the worldwide academic communities and contributes to the progress and application of science with its publication.

Other selected journals from SCIRP are listed as below. Submit your manuscript to us via either submit@scirp.org or Online Submission Portal.
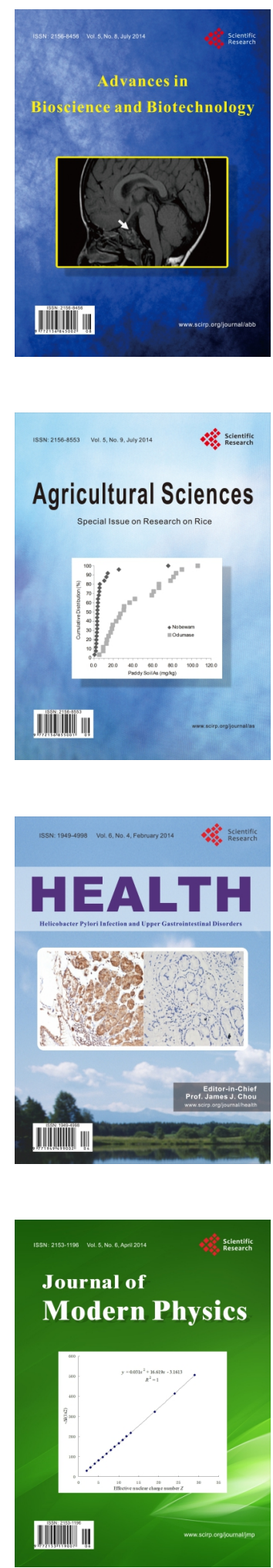
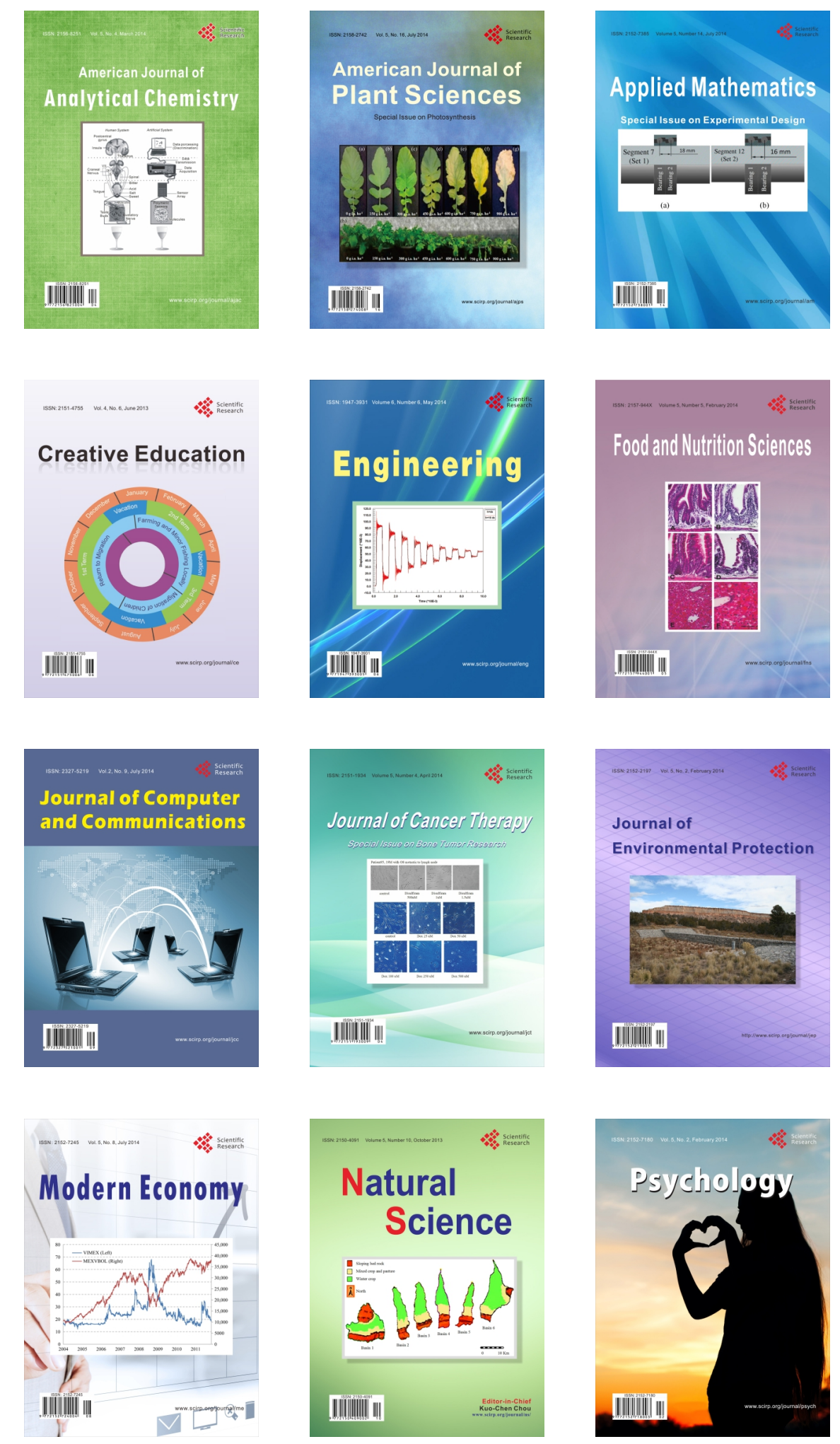